\title{
Síntese sustentável de compostos heterocíclicos. Azaindole - um núcleo privilegiado em química medicinal
}

\author{
Ana Sofia Santos \\ LAQV, REQUIMTE, Departamento de Química, Faculdade de Ciências e Tecnologia, Universidade Nova de Lisboa \\ asb.santos@campus.fct.unl.pt
}

\begin{abstract}
Sustainable Synthesis of Heterocyclic Compounds. Azaindole - a privileged scaffold in medicinal chemistry - Indole and its bioisostere, azaindole, are considered privileged structures in medicinal chemistry. Thus, there is a continuous effort to develop new and more sustainable ways of attaining these structures, avoiding toxic reagents, harsh conditions and difficult methods of purification. This article describes the development of synthetic methods for azaindole synthesis using metal-catalysed reactions, as well as the use of a soluble polymer support to attain heterocyclic compounds, as a cheap, bio-compatible and easy to recover polymer.
\end{abstract}

$\mathrm{O}$ indole e o azaindole são estruturas privilegiadas em química medicinal. Por isso, existe a necessidade de desenvolver métodos sintéticos mais sustentáveis para a obtenção destas estruturas, evitando o uso de reagentes tóxicos, condições drásticas e métodos de purificação dispendiosos. Este artigo descreve o desenvolvimento de métodos para a construção do anel azaindole utilizando reações catalisadas por metais, bem como as variantes em que foi utilizado um suporte polimérico solúvel, barato, biocompatível e facilmente reciclável para preparar esses compostos heterocíclicos.

\section{Introdução}

Os compostos heterocíclicos são importantes unidades estruturais de diversos compostos bioativos e por isso existe uma crescente necessidade de encontrar novas e sustentáveis metodologias que permitam um acesso fácil a estes núcleos, bem como à sua funcionalização. O indole é uma estrutura privilegiada com potencial aplicação no campo da química medicinal e está presente na estrutura de diversos compostos que existem no nosso organismo. Compostos com esta estrutura e devidamente funcionalizados podem apresentar diversas propriedades farmacológicas nomeadamente anticancerígena, antioxidante e anti-inflamatória. A serotonina, por exemplo, é um neurotransmissor que possui na sua estrutura um anel indole (Esquema 1) [1]. Os fármacos não esteroides anti-inflamatórios têm sido largamente usados para o tratamento de doenças como osteoartrite reumatoide, artrite e dor desde que foi descoberto que eles inibem a síntese das prostaglandinas. Muitos destes fármacos, como a indometacina, por exemplo, constituem uma importante classe de agentes terapêuticos para o tratamento de doenças associadas à inflamação e à dor.

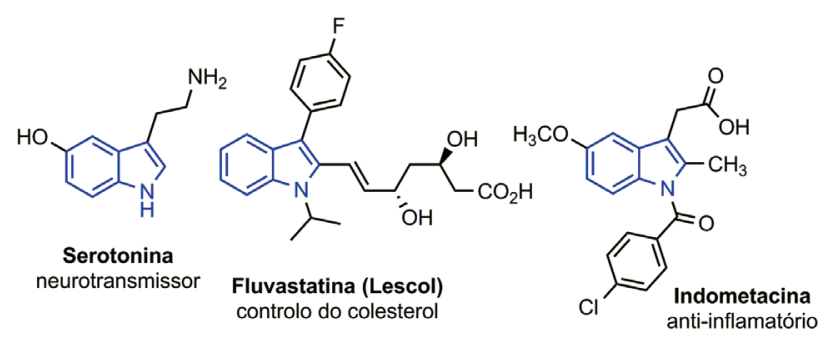

Esquema 1 - Compostos naturais e sintéticos contendo um núcleo indole.

Os azaindoles são bioisósteros do núcleo indole. São estruturas privilegiadas que têm também atraído o interesse da comunidade científica devido às suas propriedades farmacológicas. Quando devidamente funcionalizados, os azaindoles são moléculas interessantes para a descoberta de novos fármacos, visto que as suas propriedades podem ser modeladas mudando o padrão de substituição ou a posição do átomo de nitrogénio no anel (Esquema 2) [2].

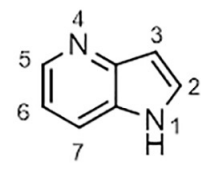<smiles>c1cc2[nH]ccc2cn1</smiles><smiles>c1cc2cc[nH]c2cn1</smiles><smiles>c1cnc2[nH]ccc2c1</smiles>

4-azaindole

5-azaindole

6-azaindole

7-azaindole

Os azaindoles são raros na natureza, mas podem ser sintetizados usando aminopiridinas como materiais de partida [3]. Dependendo do tipo de aminopiridina utilizada, podem ser obtidos diferentes isómeros deste núcleo ou diferentes padrões de substituição. Diversos compostos bioativos contendo o núcleo azaindole foram descritos recentemente [4].

\section{Síntese de azaindoles - reações catalisadas por metais de transição}

Diversos métodos sintéticos para preparar derivados de indole e de azaindole têm sido desenvolvidos, principalmente utilizando métodos de catálise metálica. Os métodos clássicos de síntese de indoles envolvem o uso de anilinas, nitrobenzenos e fenil-hidrazinas como materiais de partida [5]. Estas reações podem ser divididas em duas classes: as que usam catálise com metais de transição, como a síntese de Bartoli, e as que usam métodos clássicos, como as sínteses de Fischer e de Mandelung [6]. A abordagem mais comum para a síntese de azaindoles envolve o uso de aminopiridinas como materiais de partida. Embora esta abordagem seja semelhante à usada para a síntese de in- 
doles a partir de anilinas, a natureza eletrodeficiente do anel piridina altera as propriedades eletrónicas do sistema conjugado, o que faz com que os métodos clássicos de síntese de indole não resultem, ou não sejam tão eficientes, quando aplicados a este núcleo [7]. Por exemplo, a síntese de Fischer quando aplicada a hidrazinilpiridinas não resulta exceto quando estas possuem um grupo eletrodoador, formando-se, nesses casos, 4-azaindole e 6-azaindole em rendimentos moderados a bons [8-10]. Outro método clássico é a síntese de Bartoli que, quando aplicado à síntese de azaindole, requer o uso de um grande excesso de reagente de Grignard e apenas permite obter o 4-azaindole e o 6-azaindole com rendimentos baixos [11]. Sendo assim, todos estes métodos clássicos são pouco versáteis quando aplicados ao núcleo de azaindole e os rendimentos são baixos a moderados [12,13].

O uso de metais de transição como catalisadores tem vindo a revolucionar completamente a química orgânica moderna e a alterar a maneira como abordamos a construção de ligações C-C, C-N, C-O e C-S. O aparecimento de novas reações de acoplamento cruzado $\mathrm{C}-\mathrm{C}$ tem permitido a construção de moléculas novas e mais complexas. Vários metais de transição são utilizados nestas reações incluindo cobre, níquel, ferro e ruténio, mas o mais explorado é o paládio (Esquema 3) [14].

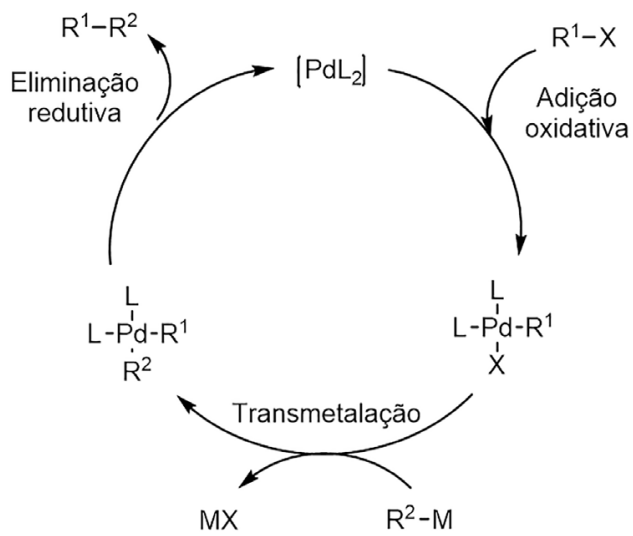

Esquema 3 - Ciclo catalítico geral para reações de acoplamento cruzado catalisadas por paládio para a formação de ligações C-C.

A crescente importância do núcleo azaindole em química biológica tem levado a um enorme desenvolvimento das metodologias para a sua preparação. As reações de acoplamento cruzado catalisadas por metais de transição são, relativamente aos métodos clássicos, uma alternativa promissora para a síntese de azaindoles.

O prémio Nobel da Química de 2010, atribuído a E. Negishi, R. Heck e A. Suzuki, contribuiu para o fortalecimento da importância da formação direta de ligações C-C através de métodos envolvendo catálise metálica [15]. Estes métodos têm vindo a transformar os procedimentos utilizados para a construção e funcionalização de uma enorme variedade de compostos importantes [4].

As reações de acoplamento cruzado catalisadas por metais de transição têm sido cada vez mais usadas na síntese de azaindoles, apesar de algumas limitações devido à capacidade de coordenação do átomo de nitrogénio da piridina com os metais [16]. Diversas vias sintéticas têm sido reportadas para a preparação destas estruturas, incluindo a reação de Sonogashira, do tipo Larock, a reação de Heck e o acoplamento de Suzuki, bem como outras reações catalisadas por paládio, incluindo os protocolos mais recentes de Cacchi e Lautens (Esquema 4) [17,18].

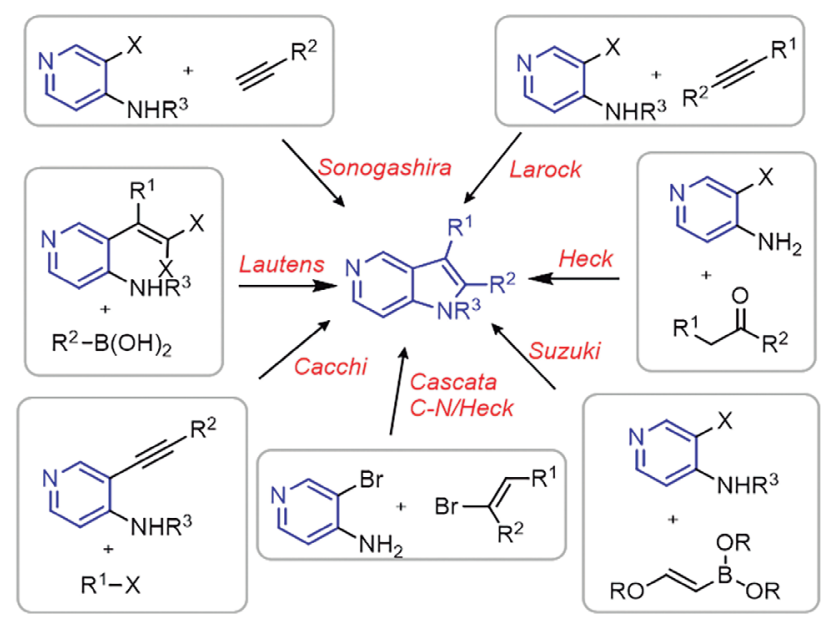

Esquema 4 - Representação de diversas reações catalisadas por metais de transição para a síntese de azaindoles.

\subsection{Reação de Sonogashira}

Os métodos de catálise metálica incluem a reação de Sonogashira, a qual foi estabelecida como um método para preparação de arilalcinos via acoplamento de alcinos terminais e haletos de arilo ou de alquilo catalisado por paládio [19]. As aminopiridinas halogenadas reagem com alcinos terminais por acoplamento de Sonogashira e esta reação é normalmente seguida da formação de um anel, usando uma base forte, como hidreto de potássio [20], ou através de ciclização mediada por cobre (Esquema 5) [21].<smiles>[R]C#Cc1cnccc1N</smiles>

Esquema 5 - Esquema geral para a síntese de azaindoles por acoplamento de Sonogashira seguido de fecho de anel.

Person [22] efetuou diversas reações de Sonogashira com aminopiridinas nitradas usando uma ciclização mediada por cobre e assistida por micro-ondas. O primeiro passo envolveu a reação de Sonogashira da 2-amino-3-iodo-5-nitropiridina com TMSA numa mistura de THF/ dimetilacetamida (DMA), seguida de ciclização para originar o azaindole desejado usando CuI em quantidade catalítica e sob irradiação com micro-ondas (Esquema 6). Este protocolo permitiu obter o azaindole com $75 \%$ de rendimento em apenas 30 minutos.

\subsection{Reação do tipo Heck}

A primeira aplicação da reação de Heck envolvendo o acoplamento de alcenos com diversas aminopiridinas halo- 


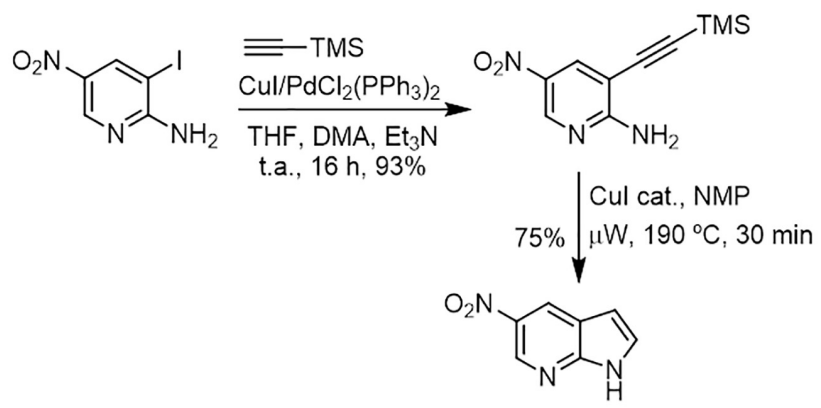

Esquema 6 - Síntese de 5-nitro-7-azaindole via reação de Sonogashira [22].

-substituídas foi reportada nos anos 90 [23]. A síntese de azaindoles por este método envolve a reação, catalisada por paládio, de amino-orto-halopiridinas com um aldeído ou uma cetona seguida de reação de Heck (Esquema 7A).

Em 2004, Nazaré e colaboradores reportaram uma ciclização catalisada por paládio de amino- orto-cloropiridinas com cetonas cíclicas e acíclicas através de formação in situ de enamina seguida de reação de Heck. Depois da otimização do método, os melhores resultados foram obtidos quando se usou $\mathrm{K}_{3} \mathrm{PO}_{4}$, $\mathrm{HOAC}$ e $\mathrm{Pd}\left(\mathrm{tBu}_{3} \mathrm{P}\right)_{2}$ em DMA a $140{ }^{\circ} \mathrm{C}$, levando à síntese de diversos derivados importantes de azaindole (Esquema 7B) [24].

A
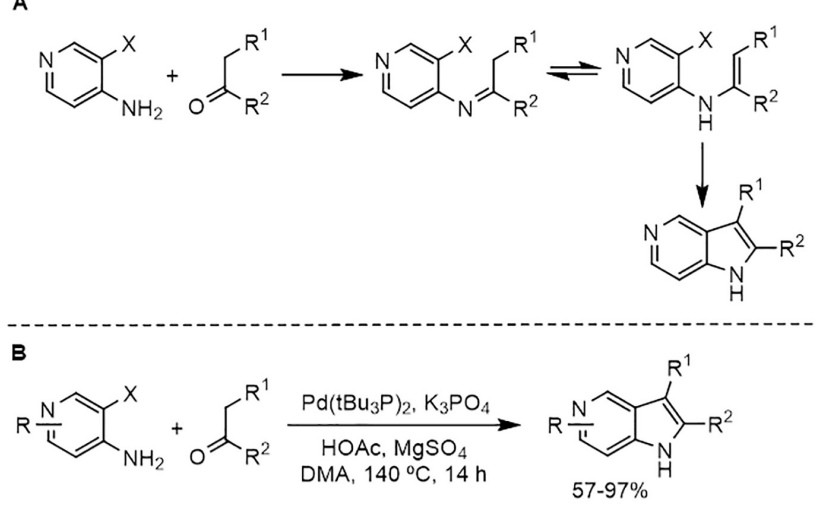

Esquema 7 - Esquema geral da síntese de azaindoles com formação in situ de enaminas e reação intramolecular de Heck (A) e ciclização catalisada por paládio de orto-amino-cloropiridinas e cetonas (B) [24].

\subsection{Reação do tipo Larock}

A ciclização catalisada por paládio de alcinos foi publicada em 1991 por Larock [25] como um método para síntese de indoles, mas tem sido largamente aplicada à preparação de azaindoles. O mecanismo desta reação para a síntese de azaindoles envolve os seguintes passos no ciclo catalítico: (a) redução do $\operatorname{Pd}(\mathrm{II})$ a $\operatorname{Pd}(0)$; (b) coordenação do halogéneo ao paládio; (c) adição oxidativa do haleto de arilo ao $\operatorname{Pd}(0)$; (d) coordenação do alcino ao paládio e subsequente inserção syn regiosseletiva na ligação arilpaládio; (d) deslocamento do nitrogénio do haleto e, por fim, (f) eliminação redutiva de forma a formar o núcleo de azaindole [26]. A regiosseletividade desta reação depende da diferença entre $\mathrm{R}^{1}$ e $\mathrm{R}^{2}$, bem como do grupo protetor $\mathrm{R}$ no grupo amino (Esquema 8A) [4].

Em 1996, Ujjainwalla e colaboradores publicaram uma nova metodologia para a síntese regiosseletiva de 5-, 6- e

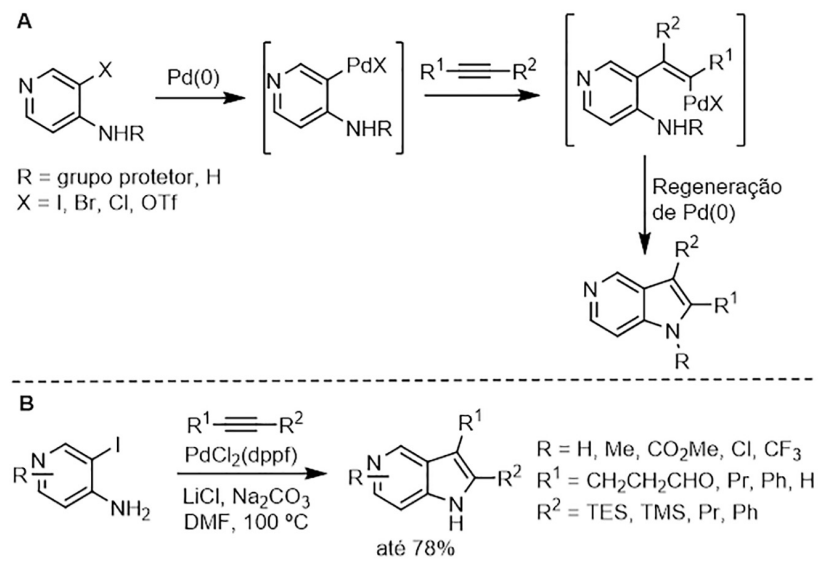

Esquema 8 - Esquema geral da ciclização tipo-Larock para síntese de azaindoles (A) e ciclização tipo-Larock regiosselectiva para síntese de 5-, 6- e 7-azaindoles (B) [25].

7-azaindoles. Usando amino-iodopiridinas desprotegidas e alcinos alil/silil- e alquil/alquil-dissubstituídos, na presença de $\mathrm{PdCl}_{2}$ (dppf), $\mathrm{LiCl}$ e $\mathrm{Na}_{2} \mathrm{CO}_{3}$ em DMF, obtiveram os respetivos azaindoles 2,3-dissubstituídos com bom rendimento (Esquema 8B) [27].

Os procedimentos e abordagens descritos anteriormente representam um enorme avanço na procura por novas e melhores maneiras de sintetizar núcleos de azaindole. Ainda assim existem algumas desvantagens. Alguns destes métodos ainda possuem baixa regiosseletividade e são limitados a poucos isómeros, diminuindo assim a versatilidade da reação. Frequentemente são necessárias condições drásticas, a prévia N-proteção da aminopiridina e a presença de grupos eletroatratores mas, ainda assim, estes são métodos efetivos para a síntese de azaindoles. Apesar disso, existe a necessidade de estudar e melhorar estas reações de modo a encontrar métodos de síntese que sejam inovadores e que exijam condições menos drásticas [4].

\subsection{Reação em cascata de acoplamento cruzado C-N/reação de Heck}

Em 2016, o nosso grupo publicou um método prático para sintetizar azaindoles a partir de amino-orto-bromopiridinas e bromoalcenos (Esquema 9) [3]. Esse método consiste numa reação em cascata catalisada por paládio envolvendo de acoplamento cruzado C-N/reação de Heck. O primeiro passo consistiu numa reação de acoplamento cruzado C-N de amino-orto-bromopiridinas com bromoalcenos, envolvendo a formação in situ de um intermediário imina/enamina seguido de reação de Heck, para originar os núcleos de azaindole. A reação em cascata torna possível a realização de duas transformações químicas num mesmo passo, evitando o isolamento complicado de intermediários. O sistema de $\mathrm{Pd}_{2}(\mathrm{dba})_{3} / \mathrm{XPhos} / \mathrm{NaOtBu}$ é eficiente para a síntese de 4-, 5-, 6- e 7-azaindoles substituídos. Apesar de ser adequado para preparar azaindoles 2-substituídos, este protocolo não se aplica a $\mathrm{N}$-arilamino-orto-bromopiridinas, o que limita o acesso a 1,2-diaril-azaindoles. Procedimentos alternativos para sintetizar esses compostos estão a ser investigados evitando a difícil $\mathrm{N}$-arilação de azaindoles 2-substituídos [28-30]. 


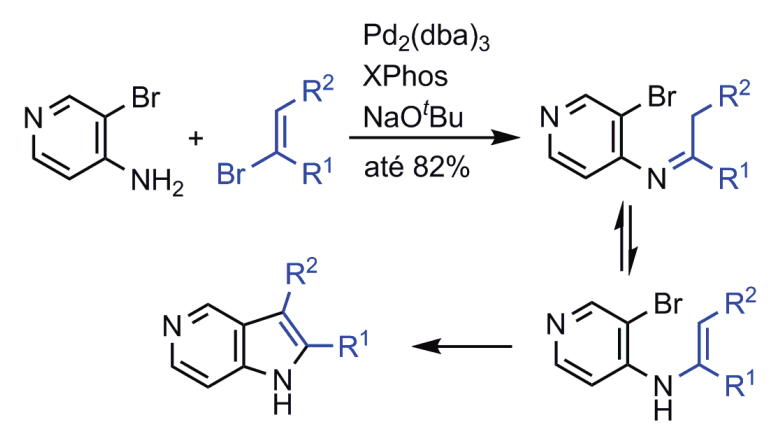

Esquema 9 - Esquema geral da cascata de aminação de bromoalcenos/ reação de Heck com amino-orto-bromopiridinas [3].

\section{PEG como suporte para síntese de indole}

O interesse crescente em reações de acoplamento cruzado catalisadas por paládio ou cobre tem levado ao desenvolvimento de versões mais sustentáveis dessas reações. Em particular o polietilenoglicol (PEG) tem sido largamente explorado como solvente e suporte em reações desse tipo (Figura 1) [31].

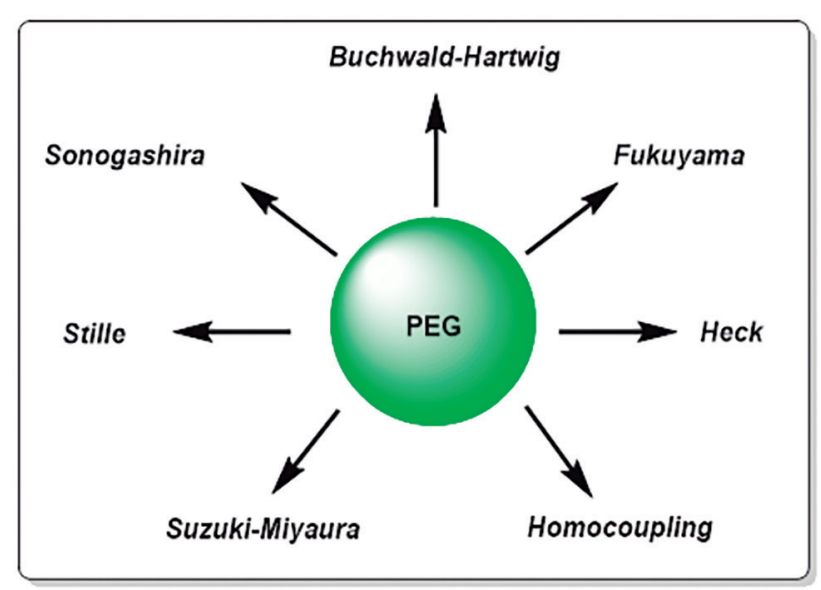

Figura 1 - Utilização de PEG como suporte e solvente em diversas reações catalisadas por metais de transição.

O uso de PEG como solvente alternativo, ou como suporte, tem vindo a crescer. Este polímero está disponível numa larga gama de pesos moleculares, é estável termicamente, não é tóxico, é barato e recuperável. O PEG surge, portanto, como uma alternativa mais amiga do ambiente relativamente a outros solventes orgânicos [31].

As reações de acoplamento cruzado catalisadas por metais de transição são conhecidas como ferramentas essenciais em química orgânica para a síntese de certos núcleos. Contundo, estes métodos requerem muitas vezes condições drásticas, como temperaturas altas, bem como o uso de solventes tóxicos. O PEG tem sido usado com sucesso em diversas reações catalisadas por metais de transição e é descrito como sendo um meio mais barato e eficiente, melhorando os rendimentos das reações e evitando purificações complicadas.

No nosso trabalho procurámos melhorar a compatibilidade entre reações de acoplamento cruzado catalisadas por metais de transição e o uso de um suporte polimérico solúvel para imobilização de substratos, de modo a facilitar a purificação dos produtos obtidos. Essa via foi usada para a preparação de compostos heterocíclicos, em particular dos tipos indole e azaindole [3,4,32].

Em 2016 o nosso grupo publicou um novo método de síntese de azaindoles envolvendo uma reação de acoplamento cruzado C-N/reação de Heck e usando amino-orto-halopiridinas e bromoalcenos como materiais de partida [3].

Dadas as dificuldades associadas à síntese de azaindoles, o desenvolvimento de um protocolo baseado em PEG2000, combinado com reações catalisadas por metais de transição, representou um enorme avanço na sustentabilidade e versatilidade da via sintética para azaindoles e o seu isolamento.

\section{Perspetivas futuras}

Pretende-se continuar a otimização das condições de síntese de indoles e azaindoles através de reações catalisadas por metais de transição usando PEG-2000 como suporte polimérico solúvel. Os avanços alcançados irão facilitar a síntese e purificação destes compostos e levar ao desenvolvimento de métodos mais simples e sustentáveis para obter estas estruturas privilegiadas.

\section{Agradecimentos}

À minha orientadora, Prof. Maria Manuel Marques, por todo o apoio e contínuo entusiasmo que tanto me ajudaram e inspiraram neste trabalho. Um agradecimento especial aos meus companheiros de laboratório.

\section{Referências}

[1] M.S. Estevão, L.C. Carvalho, D. Ribeiro, D. Couto, M. Freitas, A. Gomes, L.M. Ferreira, E. Fernandes, M.M.B. Marques, Eur. J. Med. Chem. 45 (2010) 4869-4878.

[2] J.Y. Mérour, F. Buron, K. Plé, P. Bonnet, S. Routier, Molecules 19 (2014) 19935-19979.

[3] M.J.D. Pires, D.L. Poeira, S.I. Purificação, M.M.B. Marques, Org. Lett. 18 (2016) 3250-3253.

[4] M.J. Dias Pires, D.L. Poeira, M.M.B. Marques, Eur. J. Org. Chem. (2015) 7197-7234.

[5] D.F. Taber, P.K. Tirunahari, Tetrahedron 67 (2011) 71957210.

[6] M. Inman, C.J. Moody, Chem. Sci. 4 (2013) 29-41.

[7] J.J. Song, J.T. Reeves, F. Gallou, Z. Tan, N.K. Yee, C.H. Senanayake, Chem. Soc. Rev. 36 (2007) 1120.

[8] F.G. Mann, A.F. Prior, T.J. Willcox, J. Chem. Soc. (1959) 3830-3834.

[9] R.A. Abramovitch, K.A.H. Adams, Can. J. Chem. 40 (1962) 864-869.

[10] A.H. Kelly, J. Parrick, J. Chem. Soc. C Org. (1970) 303307.

[11] Z. Zhang, Z. Yang, N.A. Meanwell, J.F. Kadow, T. Wang, J. Org. Chem. 67 (2002) 2345-2347.

[12] F. Popowycz, J.Y. Mérour, B. Joseph, Tetrahedron 63 (2007) 8689-8707.

[13] L. Guandalini, E. Martini, F. Gualtieri, M.N. Romanelli, K. Varani, Arkivoc (2004) 286-300.

[14] K.C. Nicolaou, P.G. Bulger, D. Sarlah, Angew. Chem. Int. Ed. 44 (2005) 4442-4489.

[15] C.C.C.J. Seechurn, M.O. Kitching, T.J. Colacot, V. Snieckus, Angew. Chem. Int. Ed. 51 (2012) 5062-5085.

[16] I. Nakamura, Y. Yamamoto, Chem. Rev. 104 (2004) 21272198. 
[17] S. Cacchi, G. Fabrizi, L.M. Parisi, J. Comb. Chem. 7 (2005) 510-512.

[18] Y.Q. Fang, J. Yuen, M. Lautens, J. Org. Chem. 72 (2007) 5152-5160.

[19] K. Sonogashira, Y. Tohda, N. Hagihara, Tetrahedron Lett. 16 (1975) 4467-4470.

[20] C. Koradin, W. Dohle, A.L. Rodriguez, B. Schmid, P. Knochel, Tetrahedron 59 (2003) 1571-1587.

[21] J.A. Dority, E.R. Bacon, G.Y. Lesher, V. Kumar, B. Singh, J. Org. Chem. 57 (1992) 6995-6998.

[22] S.E. Pearson, S. Nandan, Synthesis (2005) 2503-2506.

[23] M. Mori, K. Chiba, Y. Ban, Tetrahedron Lett. 18 (1977) 1037-1040.

[24] M. Nazaré, C. Schneider, A. Lindenschmidt, D.W. Will, Angew. Chem. Int. Ed. 43 (2004) 4526-4528.
[25] R.C. Larock, E.K. Yum, J. Am. Chem. Soc. 113 (1991) 6689-6690.

[26] Z. Wang, Compr. Org. Name React. Reagents, 2010, 1717-1721.

[27] F. Ujjainwalla, D. Warner, Tetrahedron Lett. 39 (1998) 5355-5358.

[28] S.I. Purificação, M.J.D. Pires, R. Rippel, A.S. Santos, M.M.B. Marques, Org. Lett. 19 (2017) 5118-5121.

[29] C.S. Hong, J.Y. Seo, E.K. Yum, Tetrahedron Lett. 48 (2007) 4831-4833.

[30] G. Qian, X. Hong, B. Liu, H. Mao, B. Xu, Org. Lett. 16 (2014) 5294-5297.

[31] M.J.D. Pires, S.I. Purificação, A.S. Santos, M.M.B. Marques, Synthesis 49 (2017) 2337-2350.

[32] L.C.R. Carvalho, M.J.D. Pires, E. Fernandes, M.M.B. Marques, RSC Adv. 3 (2013) 25711-25715.

\section{Atualidades Científicas}

\section{Dicarboxilação de 1,3-dienos com $\mathrm{CO}_{2}$}

O dióxido de carbono é um poluente, mas também pode ser uma matéria-prima útil. Os últimos anos têm testemunhado um interesse crescente por técnicas catalíticas que promovam a sua utilização na formação de compostos com valor acrescentado. Por exemplo, a incorporação de $\mathrm{CO}_{2}$ em olefinas em reações de carboxilação pode levar à formação de ácidos carboxílicos industrialmente relevantes. Apesar dos avanços realizados, os protocolos de carboxilação existentes estão confinados à inserção de uma única molécula de $\mathrm{CO}_{2}$. A possibilidade de inserção de múltiplas moléculas de $\mathrm{CO}_{2}$ abriria o caminho a novas estratégias para a preparação de ácidos policarboxílicos saturados. No entanto, a inserção múltipla de $\mathrm{CO}_{2}$ tem sido problemática.

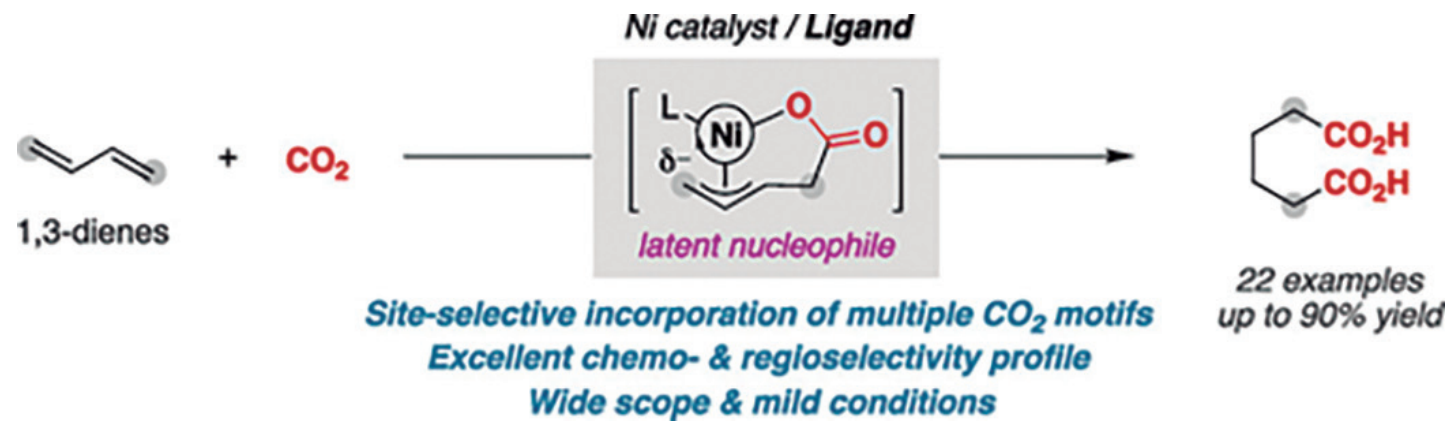

R. Martin e colegas, do Instituto de Ciência e Tecnologia de Barcelona (Tarragona, Espanha), desenvolveram um protocolo para inserir duas moléculas de $\mathrm{CO}_{2}$ em ligações duplas $\mathrm{C}=\mathrm{C}$ de dienos conjugados. A reação é catalisada por níquel, ocorre em condições suaves e apresenta elevada seletividade. Os melhores resultados foram obtidos usando como catalisador o sistema NiBr4(TBA)2/4,7-difenil-2-metil-1,10-fenantrolina (TBA = tetrabutilamónio) e Mn como redutor, na presença de $\mathrm{CO}_{2}(1 \mathrm{~atm})$ em dimetilacetamida a $50{ }^{\circ} \mathrm{C}$. Este protocolo permitiu a síntese de derivados de ácido adípico com bons rendimentos, elevada regio- e quimiosseletividade. O método é tolerante a vários grupos funcionais.

\section{Fontes:}

Dicarboxylation with $\mathrm{CO}_{2}$, http://www.chemistryviews.org/details/news/10844157/Dicarboxylation_with_CO2.html (Acedido em 05/02/2018)

A. Tortajada, R. Ninokata, R. Martin. Ni-catalyzed site-selective dicarboxylation of 1,3-dienes with $\mathrm{CO}_{2}$. J. Am. Chem. Soc. 140 (2018) 2050-2053.
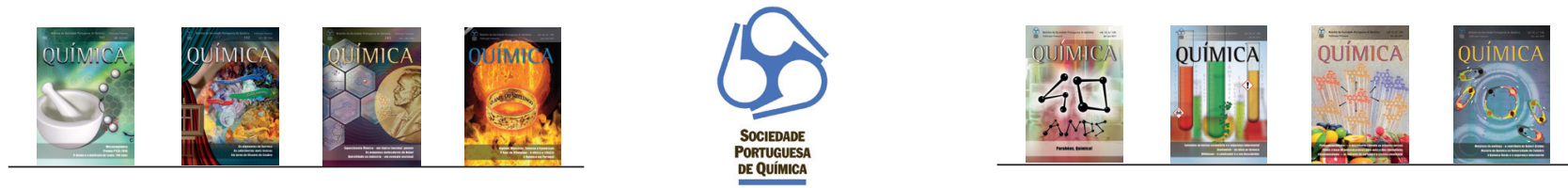